\title{
Rapid chiral separation and impurity determination of ropivacaine and bupivacaine by Densitometry-HPTLC, using mucopolysaccharide as chiral mobile phase additive
}

\author{
Nahla Nour El-Din Salama* \\ Pharmaceutical Chemistry, Department National Organization for Drug Control and Research, Giza, 35521, Egypt \\ *Corresponding author at: Pharmaceutical Chemistry, Department National Organization for Drug Control and Research, Giza, 35521, Egypt. \\ Tel.: +2.02.35851299. Fax: +2.02.35855582. E-mail address: salama nahla2004@hotmail.com (N.N.E.A. Salama).
}

\section{ARTICLE INFORMATION}

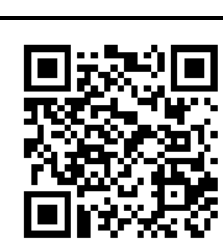

DOI: 10.5155 /eurjchem.5.2.214-218.964

Received: 02 November 2013

Received in revised form: 27 November 2013

Accepted: 28 November 2013

Online: 30 June 2014

\section{KEYWORDS}

\section{Validation}

2,6-Dimethylaniline

Densitometry-HPTLC

Ropivacaine enantiomers

Bupivacaine enantiomers

Chiral mobile phase additive

\section{Introduction}

It is well known that for chiral drugs, pharmaceutical activity resides mostly in one of the enantiomers, and unwanted side effects or even toxic effects are often observed for the other enantiomer [1]. From this reason, the separation of enantiomers is an important subject in pharmaceutical analysis. Bupivacaine is a potent local anaesthetic; has been marketed as a 50:50 racemic mixture of two enantiomers [2]. The molecular structure of this highly lipid soluble and protein bound compound contains a chiral centre in the piperidine ring, resulting in two optically active stereoisomers. Accordingly; the new long-acting local anaesthetics, ropivacaine and levobupivacaine have been developed as safer alternatives to bupivacaine. Ropivacaine and Levobupivacaine are $S(+)$ enantiomers of two different molecules, 1-propyl-2'6'pipecoloxylidide and 1-butyl-2',6'-pipecoloxylidide, respectively $[3,4]$ and their potential organic impurity 2,6-DMA are shown in Figure 1.

The mechanism of bupivacaine induced cardiac arrhythmias may result from its inhibitory effect on sodium channel current. The bupivacaine induced block of the inactivated state of sodium channels displayed stereo selectivity with $R(-)$ enantiomer interacting faster and more potently. Lower potency of $S(+)$ bupivacaine to block a particular subset of cardiac sodium channels might explain its lower cardio toxicity $[5,6]$.

Different analytical methods have been developed for separation of enantiomers. Chiral separation by capillary electrophoresis (CE) using a variety of chiral selectors have been reported [7-10]. Among them cyclodextrins and their derivatives are the most frequently employed [11]. Recently mucopolysaccharides have also been found to be effective as chiral selectors in CE $[12,13]$. These substances have only low absorbance in the UV region, which is beneficial for high detection sensitivity. Methods based on high performance liquid chromatography (HPLC) using chiral stationary phases have been most widely used for analysis of both drug enantiomers in pharmaceutical preparations and biological fluids [14-17]. Various kinds of columns have been used. Moreover derivatives CDs impregnated to silica gel normal TLC plates were reported for chiral separation of both drugs $[18,19]$. In the literature, there is no reference for direct enantiomeric resolution and determination of Rop, Bup and their chiral $(-) R$ and potential organic impurities $(2,6-D M A)$ using mucopolysaccharide as chiral mobile phase additive.

The present work describes direct and economic HPTLCdensitometric method for enantioseparation and determination 
of (-) R- and (+) S-Rop- $\mathrm{HCl}$ and $B u p-\mathrm{HCl}$ and their potential organic impurity 2,6-DMA using mucopolysacharid (Chondroitin) as chiral mobile phase additive at different temperatures. Moreover iodine vapor and UV lamp were used for the detection of chiral and organic impurities of both drugs. This method may become the method of choice compared with other techniques for economic fast routine analysis of both drugs.

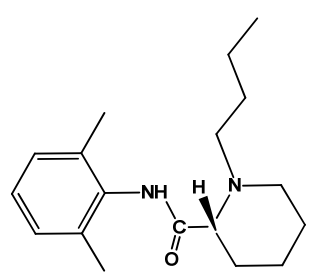

$R$-Bupivacaine

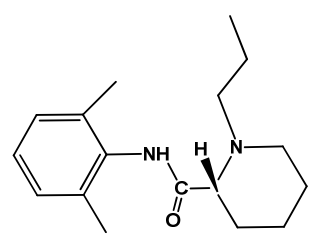

$R$-Ropivacaine

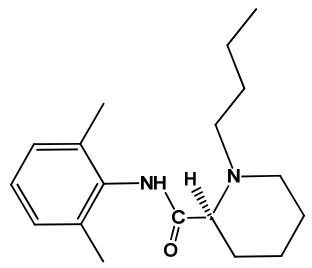

S- Bupivacaine

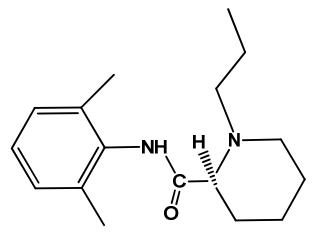

S-Ropivacaine

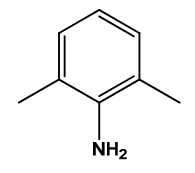

2,6-Dimethyl aniline

Figure 1. The chemical structure of $(R)$ - and $(S)$-bupivacaine, ropivacaine and their potential organic impurity 2,6-dimethyl aniline.

\section{Experimental}

\subsection{Chemicals and reagents}

All chemicals were of analytical grade if not stated otherwise. (+)S-Ropivacaine and (-)R-ropivacaine were kindly supplied by Asra-Zenica, Co., UK. Naropin vial were labeled to contain $7.5 \mathrm{mg} / \mathrm{mL}(S)$-ropivacaine per vial was purchased from the market. $(+) S$-Bupivacaine and $(-) R$-bupivacaine were kindly supplied by Astra-Zenica, Co. UK. Bucain vial were labeled to contain $0.5 \%$ bupivacaine per vial (Delta select, Co., Egypt) was purchased from the market. 2,6-Dimethylaniline (98.00\%) was purchased from Merck Schuchardt OHG, Co., Germany. Chondroitin sulfate (Bovine) BN 20/20226 was kindly supplied from Unipharma Co. Egypt. Methanol, acetonitrile and dichloromethane were obtained from Lab. Scan, Ireland.

\subsection{Equipment}

HPTLC plates $\left(20 \times 20 \mathrm{~cm}^{2}\right.$, aluminium plates precoated with $0.2 \mathrm{~mm}$ Nano-Silica gel 60 with fluorescence indicator $\mathrm{F}_{254}$ were purchased from Merck, Co., Germany. The samples were applied to the plates with $25 \mu \mathrm{L}$ Hamilton microsyringe. UV short wavelength $(254 \mathrm{~nm})$ lamp (Desaga, Germany) and Shimadzu dual wavelength flying spot densitometer, Model CS9301, PC were used. The experimental conditions of the measurements were as follows: wavelength $=220 \mathrm{~nm}$ for each $(-) R$ - and $(+) S$ - enantiomers and 2,6-dimethylaniline, photo mode $=$ reflection, scan mode $=$ zigzag, and swing width $=10$.

\subsection{Standard solutions}

Standard stock solutions $(1 \mathrm{mg} / \mathrm{mL})$ of each $(-) R$ - and $(+) S-$ Rop- $\mathrm{HCl}, \mathrm{Bup}-\mathrm{HCl}$ enantiomers, and 2,6-(DMA) were prepared by dissolving appropriate amounts of each in methanol. The stock solutions were subsequently used to prepare working standards in concentration ranges of $0.10-1.00 \mathrm{mg} / \mathrm{mL}$ for each single isomer $(-) R$ - and $(+) S-$, and $0.10-0.80 \mathrm{mg} / \mathrm{mL}$ of potential organic impurity by further dilution with methanol.

\subsection{Chromatographic conditions}

Cleaned, dried and paper-lined glass chambers $(12 \times 24 \times 24$ $\mathrm{cm})$ were used for developing chromatograms. They were preequilibrated with developer for 10 minutes. The normal TLC plates were prepared by running the mobile phase consisting of, acetonitrile:water:methanol in ratio of $(16: 3: 1, v: v: v)$ containing $0.25 \%$ ( $w: v)$ of chiral selector (chondroitin) in the usual ascending way and air dried. For detection and quantification, $20 \mu \mathrm{L}$ of each racemic solution and $10 \mu \mathrm{L}$ of their single isomer $(-) R^{-}$and $(+) S-$, and 2,6-DMA of different concentrations within the quantification range were applied side-by-side as separate compact spots $20 \mathrm{~mm}$ apart and 10 $\mathrm{mm}$ from the bottom of the HPTLC plates using a $25 \mu \mathrm{L}$ Hamilton micro syringe. The chromatograms were developed up to $8 \mathrm{~cm}$ in the usual ascending way. The plates were visualized at $254 \mathrm{~nm}$ and scanned for $R$ and $S$ isomers and 2,6$D M A$ at $220 \mathrm{~nm}$ for both drugs, by using the instrumental parameters mentioned above.

\subsection{Synthetic mixtures}

Synthetic mixtures of; $(-) R$ - and (+)S-, and 2,6-DMA within the quantification range of each drug were prepared and chromatographed according to the procedure mentioned above.

\subsection{Sample preparation}

Equivalent milliliters of each Naropin or Bucain vial equivalent to $50 \mathrm{mg}$ of $(+) S$-Rop or Bup, respectively, were extracted with dichloromethane. Aliquots of $10 \mathrm{~mL}$ of each were transferred to two beakers, air dried and transferred quantitatively to $10 \mathrm{~mL}$ volumetric flasks and diluted with methanol. Then the procedure was completed as described for the construction of calibration graphs.

\subsection{Method validation}

\subsubsection{Linearity of $(-) R-,(+) S$-enantiomers and 2,6-DMA}

Linearity was assessed by preparing six calibration sample solutions of each isomer and 2,6-DMA in the concentration range of $0.100-1.00 \mathrm{mg} / \mathrm{mL}$ in methanol and five calibration of organic impurity in concentration range of, $0.100-0.80 \mathrm{mg} / \mathrm{mL}$ in methanol for working solutions.

Regression curves were obtained by plotting peak areas versus concentration, using the least squares method. Linearity was checked for three consecutive days in the same concentration range from the same stock solutions.

\subsubsection{Limits of detection and quantification}

The limit of detection (LOD) represents the concentration of analyte that would yield a signal-to-noise ratio of three. The limit of quantification (LOQ) represents the lowest concentration of calibration curve [20,21].

The precision of the developed chiral method for each $(-) R-$ and $(+) S$ - enantiomers at limit of quantification was checked by 
Table 1. Effect of mobile phase on resolution of the enantiomers of $(R S)$-ropivacaine and (RS)-bupivacaine hydrochloride.

\begin{tabular}{|c|c|c|c|c|c|c|}
\hline \multirow{2}{*}{$\begin{array}{l}\text { Mobile phase } \\
\mathrm{CH}_{3} \mathrm{CN}: \mathrm{H}_{2} \mathrm{O}: \mathrm{CH}_{3} \mathrm{OH}\end{array}$} & \multicolumn{2}{|c|}{ Rop $h R_{F}$} & \multirow[t]{2}{*}{$\mathrm{h} R_{\mathrm{F}}(R) / \mathrm{h} R_{\mathrm{F}}(S)$} & \multicolumn{2}{|c|}{ Bup $h R_{F}$} & \multirow[t]{2}{*}{$\mathrm{h} R_{\mathrm{F}}(R) / \mathrm{h} R_{\mathrm{F}}(S)$} \\
\hline & $R$ & $S$ & & $R$ & $S$ & \\
\hline $16: 3: 1$ & 60 & 41 & 1.50 & 62 & 40 & 1.55 \\
\hline $16: 4: 1$ & 84 & 60 & 1.40 & 58 & 47 & 1.23 \\
\hline $17: 4: 0$ & 51 & 36 & 1.41 & 61 & 45 & 1.36 \\
\hline
\end{tabular}

analyzing five test solutions of each enantiomer and 2,6-DMA prepared at $\mathrm{LOQ}$ and calculating the percentage of relative standard deviation of the peak area.

\subsubsection{Precision}

Method reproducibility was determined by measuring repeatability and intermediate precision (between- day precision) of retardation factor $\left(R_{F}\right)$ and peak areas for each enantiomer and 2,6-DMA. In order to determine the repeatability of the method, freshly prepared solutions of $(-) R$ and $(+) S$ - of each drug and 2,6-DMA, in triplicate at concentrations of 2, 4 and $8 \mu \mathrm{g} / \mathrm{spot}(n=9)$ was carried out. The intermediate precision was also evaluated over three days, by performing triplicate of the same concentration each day.

\subsubsection{Accuracy}

The accuracy was determined by application of standard addition technique. Synthetic mixtures of the drug products and known amount of each (-)R- and (+)S-enantiomers and 2,6$D M A$ within the quantification range were performed.

\subsubsection{Specificity}

Specificity is the ability of the method to measure the analyte response in the presence of interfering substances. For specificity determination, synthetic mixtures of each (+)S-Rop and Bup and their chiral (-)R and organic impurities 2,6-DMA were performed and the recovery percentage was determined.

\subsubsection{Robustness}

The robustness of the method is the ability of the method to remain unaffected by small changes in parameters such as mobile phase composition, temperature, concentration of chiral selector and the saturation time of mobile phase. To determine robustness of the method, experimental conditions were purposely altered and chromatographic resolution between $(-) R$ - and $(+) S$-enantiomers and their potential organic impurity were evaluated.

\subsubsection{Solution stability}

Stability of $R$ - and $S$ - isomers in solution at analyte concentration was studied by keeping the solution in tightly capped volumetric flask at room temperature on a laboratory bench for 7 days. Content of $(-) R$ - and $(+) S$ - enantiomers and 2,6-DMA were checked for 7 days interval up to the study period.

\section{Results and discussion}

\subsection{Optimization of chromatographic conditions}

Chiral analysis of $(+) S$-Rop and Bup is of great importance since only the (S)-enantiomer is used when ropivacaine is given as a drug. The amount of $(-) R$-enantiomer should be as low as possible. Using the proposed method, it was possible to detect an impurity of less than $0.6 \mu \mathrm{g} / \mathrm{spot}$ of $(-) R$-Rop and $(-) R-B u p$ present in (+)S-Rop and (+)S-Bup, respectively. The aim of this work is to develop validated chiral HPTLC method for enantioseparation and accurate quantification of both $(+) S$ - and $(-) R$ - enantiomers and their organic impurity $(2,6-D M A)$. Racemic mixture solution of $1 \mathrm{mg} / \mathrm{mL}$ prepared in methanol was used in the method. To develop rugged and suitable HPTLC method for the separation of Rop and Bup enantiomers, and 2,6-DMA, different mobile phases, chiral selectors and TLC plates at different temperatures were tried.

Several trial runs were systematically performed using different ratios of the solvent system acetonitrile-methanolwater. The successful solvent system was acetonitrile: methanol:water (16:1:3, v:v:v) containing $0.25 \%$ chondrointin as CMPA, as shown in Table 1.

The results are average of at least 12 identical runs. Normal HPTLC plates showed good resolution with $R_{F}=0.62$ and $R_{F}=$ 0.60 for $R$ - and $R_{F}=0.40$ and $R_{F}=0.42$ for S-forms for Rop and Bup, respectively, and 0.87 for their potential organic impurity 2,6-DMA as shown in Figure 2.

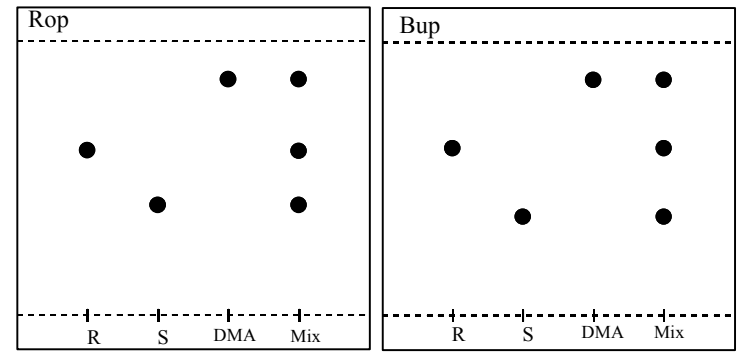

Figure 2. Thin layer chromatograms showing resolution of $(R)$ - and $(S)$ ropivacaine and bupivacaine $(10 \mu \mathrm{g} / \mathrm{spot})$, and their potential organic impurity 2,6-DMA, mobile phase acetonitrile:methanol:water (16:1:3, v:v:v) containing $0.25 \%$ chondroitin as CMPAs, Temperature: $20^{\circ} \mathrm{C}, \mathrm{pH}=6.5$.

In order to achieve the separation of ropivacaine and bupivacaine enantiomeric forms, different concentrations of chondroitin were tried in concentration range, 0.1-0.5 g\%, and the optimal concentration was achieved with $0.25 \mathrm{~g} \%$, the results are shown in Figure 3.

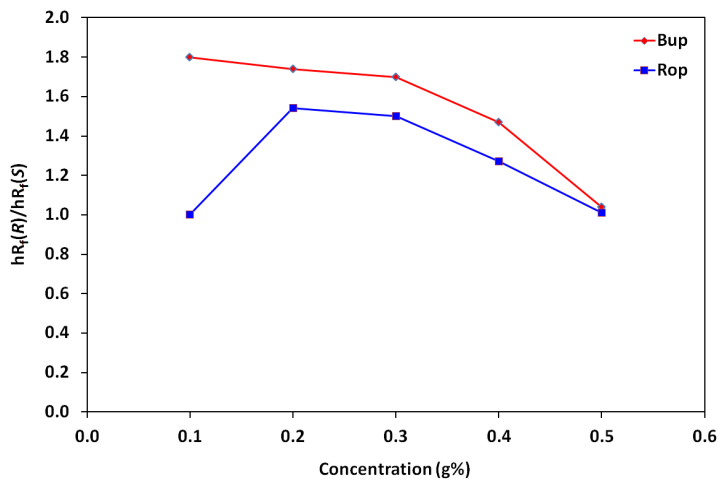

Figure 3. Effect of chondroitin concentration on enantiomeric resolution of $(R S)$-ropivacaine and $(R S)$-bupivacaine $(10 \mu \mathrm{g} / \mathrm{spot})$.

Chiral interaction between the analyte and chiral selector are known to be affected by temperature [22]. In our study different temperatures; 4,20 and $40{ }^{\circ} \mathrm{C}$ were studied. The best resolution was obtained at $20 \pm 2{ }^{\circ} \mathrm{C}$ as shown in Figure 4 . Decrease in temperature $\left(4^{\circ} \mathrm{C}\right)$ showed no significant difference, whereas increase in temperature showed a tendency for low selectivity. 
Table 2. Results from validation of the method.

\begin{tabular}{|c|c|c|c|}
\hline \multirow[t]{2}{*}{ Parameters } & \multicolumn{3}{|l|}{ Values } \\
\hline & Ropivacaine & Bupivacaine & 2,6-Dimethyl aniline \\
\hline \multicolumn{4}{|l|}{ Repeatability (RSD[\%], $\mathrm{n}=9$ ) } \\
\hline$R_{F}(R)$ enantiomer & 0.07 & 0.06 & 0.09 \\
\hline$R_{F}(S)$ enantiomer & 0.03 & 0.02 & \\
\hline Peak area $(R)$-enantiomer & 1.70 & 2.83 & 3.81 \\
\hline Peak area $(S)$-enantiomer & 2.32 & 1.71 & \\
\hline \multicolumn{4}{|l|}{ Intermediate precision (RSD [\%], $\mathrm{n}=27$ ) } \\
\hline$R_{F}(R)$-enantiomer & 0.17 & 0.16 & 0.17 \\
\hline$R_{F}(S)$-enantiomer & 0.23 & 0.21 & \\
\hline Peak area $(R)$-enantiomer & 1.70 & 2.83 & 4.81 \\
\hline Peak area $(S)$-enantiomer & 2.32 & 1.71 & \\
\hline \multicolumn{4}{|l|}{$L O D$} \\
\hline Limit of detection $[\mu \mathrm{g} / \mathrm{spot}]$ for $(R)$-enantiomer & 0.50 & 0.57 & 0.60 \\
\hline Limit of detection $[\mu \mathrm{g} / \mathrm{spot}]$ for $(S)$-enantiomer & 0.42 & 0.30 & \\
\hline \multicolumn{4}{|l|}{ LOQ } \\
\hline Limit of quantification $[\mu \mathrm{g} / \mathrm{spot}]$ for $(R)$-enantiomer & 1.00 & 1.00 & 1.00 \\
\hline Precision (RSD [\%]) & 2.35 & 3.75 & 1.59 \\
\hline Limit of quantification $[\mu \mathrm{g} / \mathrm{spot}]$ for $(S)$-enantiomer & 1.00 & 1.00 & \\
\hline Precision (RSD [\%]) & 2.77 & 2.56 & \\
\hline \multicolumn{4}{|l|}{ Linearity for $(R)$-enantiomer } \\
\hline Calibration range $[\mu \mathrm{g} / \mathrm{spot}]$ & $1-10$ & $1-10$ & $1-8$ \\
\hline Calibration points & 6 & 6 & 5 \\
\hline Correlation coefficient & 0.9993 & 0.9995 & 0.9997 \\
\hline SE of slope [\%] & 7.65 & 6.90 & 11.23 \\
\hline SE of intercept [\%] & 46.55 & 42.25 & 55.27 \\
\hline \multicolumn{4}{|l|}{ Linearity for $(S)$-enantiomer } \\
\hline Calibration range $[\mu \mathrm{g} / \mathrm{spot}]$ & $1-10$ & $1-10$ & \\
\hline Calibration points & 6 & 6 & \\
\hline Correlation coefficient & 0.9994 & 0.9994 & \\
\hline SE of slope [\%] & 8.72 & 11.36 & \\
\hline SE of intercept [\%] & 48.86 & 103.56 & \\
\hline
\end{tabular}

Table 3. Robustness of the method.

\begin{tabular}{|c|c|c|}
\hline \multirow[t]{2}{*}{ Conditions } & \multirow{2}{*}{$\begin{array}{l}\text { Ropivacaine } \\
\text { Selectivity } \\
\mathrm{h} R_{F}(R) / \mathrm{h} R_{F}(S)\end{array}$} & \multirow{2}{*}{$\begin{array}{l}\text { Bupivacaine } \\
\text { Selectivity } \\
\mathrm{h} R_{F}(R) / \mathrm{h} R_{F}(S)\end{array}$} \\
\hline & & \\
\hline \multicolumn{3}{|c|}{ Concentration of chiral selector [g\%] } \\
\hline 0.30 & 1.30 & 1.74 \\
\hline 0.25 & 2.17 & 1.78 \\
\hline 0.20 & 1.54 & 1.67 \\
\hline \multicolumn{3}{|c|}{ Mobile phase composition } \\
\hline \multicolumn{3}{|c|}{ Acetonitrile:methanol:water } \\
\hline $16: 0.8: 3$ & 1.51 & 1.51 \\
\hline $16: 1.0: 3$ & 2.17 & 1.78 \\
\hline $16: 1.2: 3$ & 1.80 & 1.40 \\
\hline \multicolumn{3}{|l|}{ Temp $\left[{ }^{\circ} \mathrm{C}\right]$} \\
\hline 23 & 1.74 & 1.70 \\
\hline 25 & 1.80 & 2.06 \\
\hline 27 & 1.70 & 1.23 \\
\hline \multicolumn{3}{|c|}{ Saturation time [min] } \\
\hline 8 & 2.17 & 1.87 \\
\hline 10 & 2.05 & 2.06 \\
\hline 12 & 2.01 & 1.90 \\
\hline
\end{tabular}

According to the experimental conditions a control stationary without CMPA spotted with the tested compounds were eluted and resulted in no resolution of racemic mixtures.

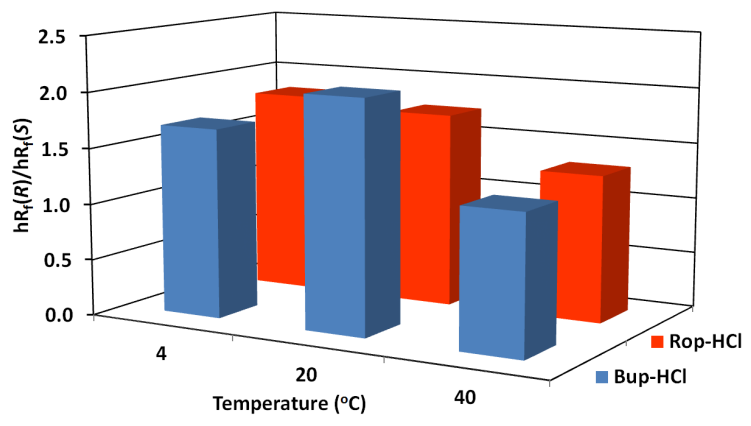

Figure 4. Effect of temperature on enantiomeric resolution of (RS)ropivacaine and $(R S)$-bupivacaine $(10 \mu \mathrm{g} / \mathrm{spot})$.

\subsection{Method validation}

In the repeatability study the relative standard deviation (RSD) for the retardation factor for $(+) S-,(-) R$-enantiomrs and 2,6-DMA and of the peak area was less than $3.0 \%$ as presented in Table 2. In the intermediate precision study, results show that RSD values were in the same order of magnitude than those obtained for repeatability as presented in Table 2 .

The limit of detection (LOD) was estimated to be 0.5 and $0.6 \mu \mathrm{g} / \mathrm{spot}$ for $(+) S$ - and $(-) R$-enantiomers, whereas limit of quantification(LOQ) was the lowest concentration of calibration curve as stated in Table 2. Good linearity was observed for $(+) S$ - and (-)R-enantiomers of both drugs over the concentration range of $1.0-10 \mu \mathrm{g} / \mathrm{spot}$ as presented in Table 2 . Linearity was checked for $(+) S$ - and (-)R-enantiomers over the same concentration range for three consecutive days. The $\%$ RSD of the slope and y-intercept of the calibration curves were shown in Table 2.

The resolution between $(+) S-,(-) R$-enantiomers and 2,6$D M A$ was greater than 1.51 under all separation conditions tested, demonstrating sufficient robustness Table 3. 
Table 4. Results of laboratory prepared mixtures Ropivacain (Conc. $\mu \mathrm{g} /$ spot)

\begin{tabular}{ll}
\hline Ratio & \\
\hline $\boldsymbol{R}$ & $\boldsymbol{S}$ \\
\hline 4 & 4 \\
1 & 8 \\
1 & 6 \\
\hline
\end{tabular}

2,6-DMA
2
1
2

Recovery $(\mathbf{n}=\mathbf{3})$
$\mathbf{0} \pm \mathrm{RSD}$ of $S$-ropivacaine
$99.50 \pm 1.04$
$101.00 \pm 1.89$
$100.75 \pm 1.77$
Bupivacaine (Conc. $\mu \mathrm{g} / \mathrm{spot}$ )

\begin{tabular}{l|l} 
Ratio \\
\hline $\boldsymbol{R}$ \\
\hline 4 \\
1 \\
1
\end{tabular}

\begin{tabular}{ll}
$\boldsymbol{S}$ & $\mathbf{2 , 6 - D M A}$ \\
\hline 4 & 2 \\
8 & 1 \\
6 & 2
\end{tabular}

Recovery ( $n=3)$ $\% \pm$ RSD of levobupivacaine $99.60 \pm 1.55$ $98.00 \pm 1.70$ $100.50 \pm 2.00$

Table 5. Application of the proposed method for determination of robivacaine and bupivacaine in their drug products and the recovery results of addition of $\mathrm{R}$ and S enantiomers.

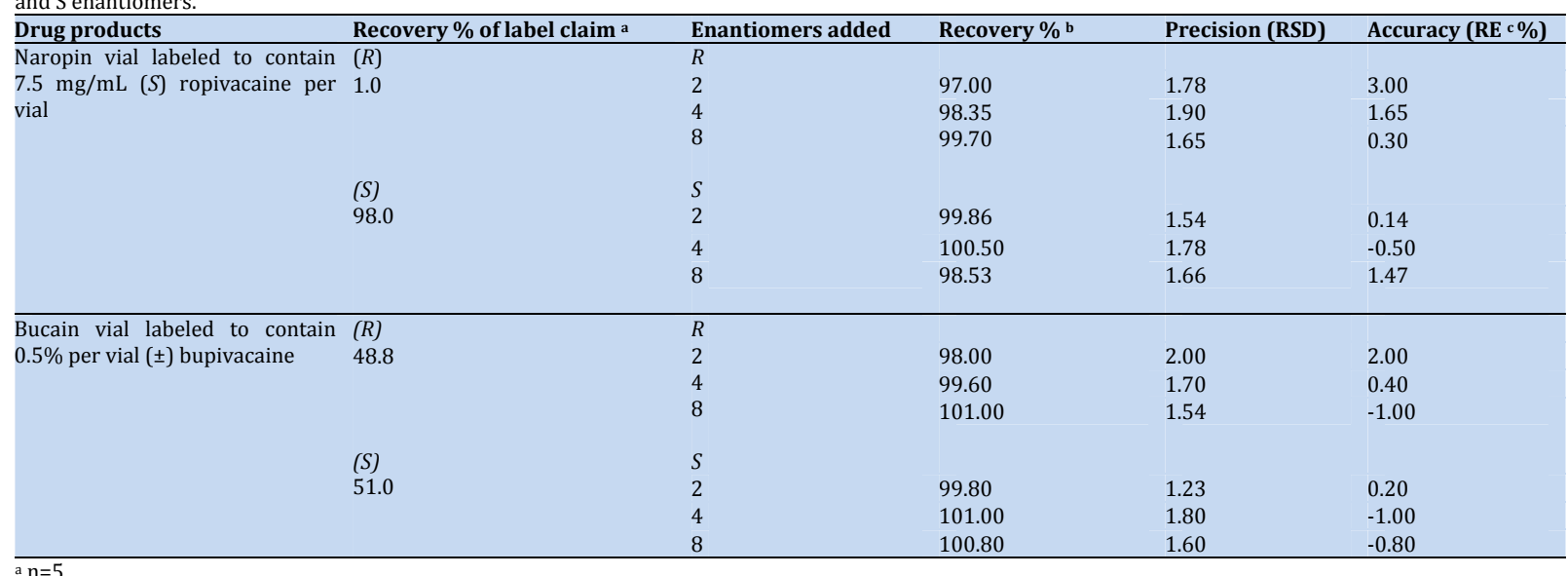

c Relative error, $\{$ (measured mean value - nominal value) $/$ nominal value $\} \times 100$.

The specificity of the method was assessed by analyzing synthetic mixtures of $(+) S$-form and its chiral $(-) R$ - and organic impurities (2,6-DMA) in different ratios as shown in Table 4.

The accuracy was assessed by applying the standard additions technique. Satisfactory results were obtained and were in good agreement with the labeled claim, as presented in Table 5 .

No significant change in the $(+) S-$ and $(-) R$ - content was observed in (Rac) Rop and (Rac) Bup samples during solution stability. Hence, (+)S-, (-)R- and 2,6-DMA samples solution are stable for at least 7 days at $25 \pm 2{ }^{\circ} \mathrm{C}$.

\section{Conclusion}

Inexpensive and environment friendly densitometricHPTLC method was described for enantioseparation and determination of Rop, Bup and their impurities; chiral (-)R- and organic (2,6-DMA). The developed method may become the method of choice, compared with other chromatographic techniques for chiral discrimination and fast routine enantiomeric purity assessment. The method was completely validated showing satisfactory data for all the method validation parameters tested.

\section{References}

[1]. Wainer, I. W.; Editor, Drug Stereochemistry: Analytical Methods and Pharmacology, 2nd Ed., Marcel Dekker, New York, 1993.

[2]. Albright, G. A. Anesthesiology 1979, 51, 285-287.

[3]. Burlacu, C. L.; Buggy, D. J. Ther. Clin. Risk. Manag. 2008, 4, 381-392.

[4]. McClure, J. H. Br. J. Anaesth. 1996, 76, 300-307.

[5]. Valenzuela, C. V.; Snyders, D. J.; Bennett, P. B.; Tamargo, J.; Hondeghem, L. M. Circulation 1995, 92, 3014-3024.

[6]. Tsuchiya, H.; Mizogami, M. Local Reg. Anesth. 2008, 1, 1-9.

[7]. Amini, A.; Javerfalk, E.; Bastami, S.; Westerlund, D. Electrophoresis 1999, 20, 204-211.

[8]. Javerfalk, E. M.; Amini, A.; Westerlund, D.; Andren, P. E. J. Mass Spectrom. 1998, 33, 183-186.

[9]. Tang, W.; Muderawan, I. W.; Siu-Choon, N. G.; Chan, H. S. O. Anal. Chim. Acta 2006, 555, 63-67.

[10]. Amini, A.; Wiersma, B.; Westerlund, D.; Paulsen-Sorman, U. Eur. J. Pharm. Sci. 1999, 9, 17-24.
[11]. Fanali, S. J. Chromatogr. A 2000, 875, 89-122.

[12]. Gubitz, G.; Schmid, M. G. Electrophoresis 2000, 21, 4112-4135.

[13]. Nishi, H. J. Chromatogr. A 1997, 792, 327-347.

[14]. Nystrom, A.; Strandberg, A.; Aspegren, A.; Behr, S.; Karlsson, A Chromatographia 1999, 50, 208-219.

[15]. Tanaka, Y.; Terabe, S. Chromatographia 1999, 49, 489-495

[16]. Schneiderman, E.; Stalcup, A. M. J. Chromatogr. B 2000, 745, 83-102.

[17]. Arvidsson, T.; Bruce, H. F.; Halldin, M. M. Chirality 1995, 7, 272-277.

[18]. Salama, N. N. J. Planar Chromatogr. Mod. TLC. 2008, 21, 441-446.

[19]. Taha, E. A. Curr. Pharm. Chem. 2007, 3, 273-277.

[20]. ICH Q2A. Validation of analytical methods definitions and terminology, in IFPMA (ed.), International Conference on Harmonization, Geneva 1994.

[21]. ICH Q2B. Validation of analytical procedure methodology, in IFPMA (ed.), International Conference on Harmonization, Geneva 1996.

[22]. Aboul-Enein, H. Y.; El-Awady, M. I.; Heard, C. H. J. Pharm. Biomed. Anal. 2003, 32, 1055-1059. 